\title{
Chest radiography in children aged 2-59 months diagnosed with non-severe pneumonia as defined by World Health Organization: descriptive multicentre study in Pakistan
}

Tabish Hazir, Yasir Bin Nisar, Shamim A Qazi, Shazia F Khan, Mujahid Raza, Shehla Zameer, Syed Asif Masood

\begin{abstract}
Objectives To evaluate the chest radiographs of children diagnosed with non-severe pneumonia on the basis of the current World Health Organization guidelines (fast breathing alone) for radiological evidence of pneumonia.

Design Descriptive analysis.

Setting Outpatient departments of six hospitals in four cities in Pakistan.

Participants 2000 children with non-severe pneumonia were enrolled; 1932 children were selected for chest radiography. Interventions Two consultant radiologists used standardised WHO definitions to evaluate chest radiographs; no clinical information was made available to them. If they disagreed, the radiographs were read by a third radiologist; the final classification was based on agreement between two of the three radiologists.

Main outcome measures Presence or absence of pneumonia on radiographs.

Results Chest radiographs were reported normal in 1519 children (82\%). Radiological evidence of pneumonia was reported in only 263 (14\%) children, most of whom had interstitial pneumonitis. Lobar consolidation was present in only 26 children. The duration of illness did not correlate significantly with the presence of radiological changes (relative risk $1.17,95 \%$ confidence interval 0.91 to 1.49 ).

Conclusion Most children diagnosed with non-severe pneumonia on the basis of the current WHO definition had normal chest radiographs.
\end{abstract}

\section{Introduction}

Acute respiratory illnesses, primarily pneumonia, are the main cause of mortality in children under 5 years in most developing countries-they result in 1.9 million deaths each year. ${ }^{1}$ To help reduce mortality from these diseases, the World Health Organization introduced case management guidelines based on simple clinical signs for diagnosing pneumonia followed by empirical treatment with antibiotics. ${ }^{2}$ Standardised case management of pneumonia has resulted in a $30-40 \%$ reduction in deaths from this disease.

For management purposes, pneumonia is subclassified according to its severity. Children with lower chest indrawing or danger signs (central cyanosis, excessive sleepiness, inability to drink, convulsions) require admission and intravenous antibiotics, whereas those who only have fast breathing can be treated at home with oral antibiotics. ${ }^{2}$ Most studies that evaluate the useful- ness of clinical signs for detecting pneumonia are hindered by the lack of a uniformly accepted criterion for diagnosing pneumonia. The WHO case management guidelines are based on studies from the 1970s and early 1980s, which evaluated the usefulness of clinical signs for diagnosing pneumonia in children. Most of these studies used chest radiographs to diagnose pneumonia, ${ }^{4-6}$ as did most studies to determine the aetiology of pneumonia. ${ }^{78}$ Many of these studies compared the sensitivity and specificity of clinical signs in children with radiographic evidence of pneumonia, and the results showed that most of these children had fast breathing.

However, some children with fast breathing do not have pneumonia. Fast breathing can be caused by malaria, hyper-reactive airway disorder, and bronchiolitis with wheeze and fast breathing. ${ }^{9-11}$ Other factors, such as fever, can also influence the respiratory rate. ${ }^{12} \mathrm{WHO}$ guidelines recommend that the respiratory rate is measured when the child is afebrile, calm, or feeding, ${ }^{2}$ but this may not always be possible, especially in a busy outpatient setting.

Concern has therefore been voiced about using fast breathing as the only clinical sign for diagnosing pneumonia in children. ${ }^{13}$ Many children diagnosed in this way may receive antibiotics unnecessarily, which could lead to increased resistance to antibiotics in the community. These issues can only be investigated by well designed aetiological studies of pneumonia. We analysed the chest radiographs of children aged 2-59 months who presented to a health facility with fast breathing and were diagnosed with non-severe pneumonia using WHO guidelines. $^{2}$

\section{Materials and methods}

We analysed the radiological data from a prospective, randomised, double blind, placebo controlled trial that compared the effectiveness of three and five days of treatment with amoxicillin in community acquired non-severe pneumonia, defined according to the WHO guidelines. The trial was carried out in the outpatient departments of six hospitals in the cities of Gilgit, Islamabad, Lahore, Multan, and Rawalpindi in Pakistan. The outcomes of this trial have been published elsewhere. ${ }^{15}$ No chest radiographs were done at the Gilgit site. All other children had chest radiographs taken at enrolment. Some of the radiographs were not readable (figure). All chest radiographs were sent to the coordinating centre at the Children's Hospital, Islamabad, and read by two paediatric radiologists (SFK and MR), who were given no clinical information. Each paediatric radiologist gave his or her assessment independently. In case of 
Table 1 Classification of parenchymal changes for diagnosis of pneumonia ${ }^{36}$

\begin{tabular}{lcc} 
Type & Subtypes \\
\hline Interstitial & & \\
\hline Alveolar & Lobar & Non-lobar \\
\hline Mixed & & \\
\hline Perihilar & Lobar & Non-lobar \\
\hline Collapse or atelectasis & Thin walled & Single \\
\hline Cavitation & & Multiple \\
\hline & Thick walled & Single \\
\hline & & Multiple \\
\hline
\end{tabular}

Miliary

Hyperinflation

Other abnormalities

disagreement chest radiographs were read by a third radiologist (SZ). Radiological findings were classified as pneumonia, bronchiolitis, and normal.

We defined radiological evidence of pneumonia as radiologically appreciable pneumonia reported by at least two of the three radiologists, who used a standardised case report form developed by WHO for classifying parenchymal changes into predefined radiological categories. ${ }^{16}$ These radiological changes were then further defined according to the quadrants in the right and left lung fields. Parenchymal changes were classified into nine categories (table 1). No radiological evidence of pneumonia was defined as normal.

\section{Data collection and analysis}

We used a set of specially designed case report forms to record data. Radiological data were entered twice and validated. EPINFO 6.04 and SPSS 11.0 software programs were used for analysis. We used the $\kappa$ statistic, which measures the degree of inter-rater agreement over and above that expected by chance, to assess agreement between individual radiologists. To compare groups, we used the $\chi^{2}$ test for categorical variables and the Student's $t$ test for continuous variables. In univariate analysis, we recoded the continuous variables, such as age and duration of illness, to categorical variables. We report estimates of relative risk, 95\% confidence intervals, and $\mathrm{P}$ values for the categorical variables but only $\mathrm{P}$ values for continuous variables.

\section{Results}

In total, 2000 children were enrolled in the study, and 1848 chest radiographs were available for assessment by radiologists. Radiologist 1 (SFK) and radiologist 2 (MR) read all the chest
Table 2 Radiological assessments of children diagnosed with non-severe pneumonia on the basis of fast breathing alone by the three radiologists. Values are number (\%)

\begin{tabular}{lccc} 
Radiological classification & $\begin{array}{c}\text { Radiologist 1 } \\
(\mathbf{n = 1 8 4 8 )}\end{array}$ & $\begin{array}{c}\text { Radiologist 2 } \\
(\mathbf{n}=\mathbf{1 8 4 8 )}\end{array}$ & $\begin{array}{c}\text { Radiologist 3 } \\
\mathbf{( n = 3 7 1 )}\end{array}$ \\
\hline Normal & $1496(81)$ & $1366(74)$ & $270(73)$ \\
\hline Pneumonia & $259(14)$ & $416(23)$ & $87(23)$ \\
\hline Bronchiolitis & $93(5)$ & $66(4)$ & $14(4)$ \\
\hline
\end{tabular}

radiographs. Radiologists disagreed on 371 radiographs, which were then analysed by a third radiologist (table 2). Radiologists 1 and 2 agreed about 1477 radiographs $(\kappa=0.46$; table 3$)$.

The final classification of the radiographs was based on agreement between two of the three radiologists. Of the 1848 chest radiographs examined, 263 (14\%) were reported as having radiological evidence of pneumonia. Lobar consolidation was present in only 26 children and most of the others had interstitial parenchymal changes. A further 66 (4\%) radiographs were thought to be consistent with the diagnosis of bronchiolitis. The remaining 1519 (82\%) radiographs were reported as normal (fig 1).

Table 4 shows the baseline characteristics of children with and without evidence of radiological pneumonia. No statistically significant differences were found. Of the 415 children who had wheeze at enrolment, $350(84 \%)$ had normal chest radiographs. The duration of illness did not correlate significantly with the presence of radiological changes (relative risk 1.17, 95\% confidence interval 0.91 to 1.49 ).

\section{Discussion}

Most children who were diagnosed with non-severe pneumonia on the basis of fast breathing had no radiological evidence of pneumonia. Chest radiographs are thought by many to be the best method for diagnosing pneumonia. ${ }^{17}$ Chest radiographs are not recommended for diagnosing pneumonia in most developing countries because the facilities are often not available. The interpretation of radiographs is difficult in young children and is affected by the radiographer's experience and the amount of clinical information available. ${ }^{18}$ Moreover, chest radiography cannot reliably distinguish between viral and bacterial pneumonia and is often unable to detect early changes of pneumonia. ${ }^{19}$ Drawbacks of chest radiography include exposure to ionising radiation, cost, the time and space used, and the need to wait for the radiograph and see the clinician again. The WHO case management guidelines therefore still recommend the use of simple clinical signs to diagnose pneumonia.

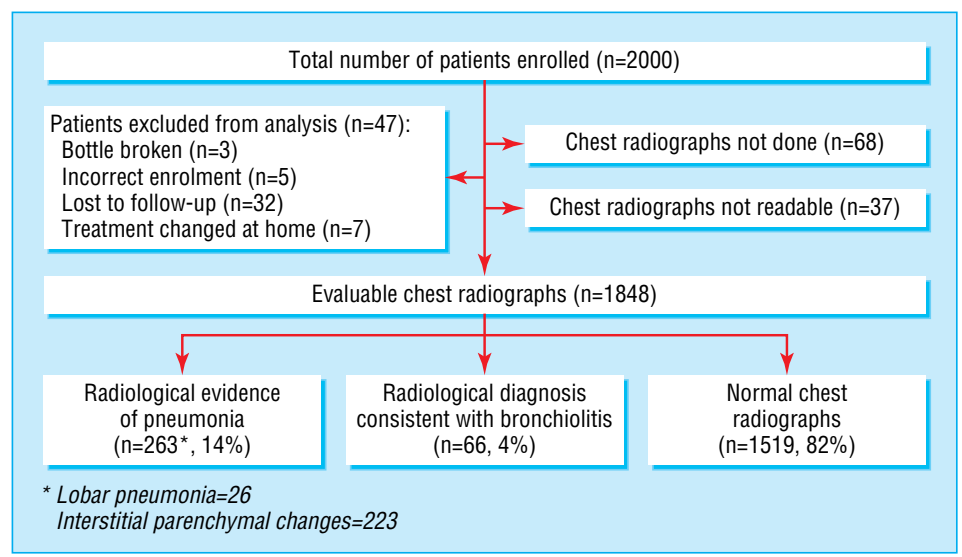

Radiological results in children diagnosed with non-severe pneumonia on the basis of fast breathing alone 
Table 3 Cross classification and interobserver agreement between two radiologists when assessing children diagnosed with non-severe pneumonia on the basis of fast breathing alone. Values are number (\%)

\begin{tabular}{lccc}
\multirow{2}{*}{ Radiologist 2 } & \multicolumn{4}{c}{ Radiologist $\mathbf{~}$} \\
\cline { 2 - 4 } & Pneumonia ( $\mathbf{n = 2 5 9 )}$ & No pneumonia $(\mathbf{n}=\mathbf{1 4 9 6})$ & Bronchiolitis (n=93) \\
\hline Pneumonia & $176(68)$ & $231(15)$ & $9(10)$ \\
\hline No pneumonia & $82(32)$ & $1252(84)$ & $32(34)$ \\
\hline Bronchiolitis & $1(0.4)$ & $13(1)$ & $52(56)$ \\
\hline$\kappa=0.46$. & & &
\end{tabular}

WHO guidelines aim to help reduce mortality from pneumonia by standardising the management of children with acute respiratory illnesses, particularly in developing countries where health workers have limited skills. These guidelines are also designed to rationalise the use of antibiotics. The severity of disease in children varies from self limiting upper respiratory tract infections that need only supportive care to disease that requires immediate referral to a well equipped health facility. In the middle are children who are not sick enough to be referred but need oral antibiotics as part of their home management. After the introduction of standard case management of acute respiratory illnesses, both a reduction in mortality and an improvement in appropriate use of antibiotics were seen. ${ }^{30}$

Current WHO definitions for classifying pneumonia need to be more specific. Some argue that chest radiographs cannot detect early changes of pneumonia and, therefore, radiographs can be normal in some children with pneumonia. Our data do not support this argument. The incidence of radiological changes was similar in children whose illness lasted longer than three days and those who presented much earlier. Our data therefore indicate that most children with normal radiographs probably did not have pneumonia.

Previous data show that a proportion of non-severe pneumonia is viral in origin, so that the use of antibiotics could be reduced by improving the specificity of WHO definitions of non-severe pneumonia. ${ }^{77}$ Fever has been identified as the best predictor of infection that requires treatment with antibiotics as children with fever were sicker, had a higher incidence of radio-

Table 4 Baseline characteristics of children diagnosed with non-severe pneumonia on the basis of fast breathing alone $(n=1782)$. Values are number $(\%)$ unless indicated otherwise

\begin{tabular}{|c|c|c|c|}
\hline \multirow[b]{2}{*}{ Characteristic } & \multicolumn{2}{|c|}{ Radiological evidence of pneumonia } & \multirow[b]{2}{*}{$\begin{array}{l}\text { Relative risk (95\% } \\
\text { CI) }\end{array}$} \\
\hline & $\begin{array}{c}\text { Present }(n=263, \\
15 \%)\end{array}$ & Absent ( $n=1519,85 \%)$ & \\
\hline \multicolumn{4}{|l|}{ Sex } \\
\hline Male & $158(60 \%)$ & $972(64 \%)$ & 0.87 (0.69 to 1.09) \\
\hline \multicolumn{4}{|l|}{ Age (months) } \\
\hline $2-5$ & $59(22 \%)$ & $391(26 \%)$ & 0.83 (0.63 to 1.11$)$ \\
\hline 6-11 & $73(28 \%)$ & $428(28 \%)$ & 0.92 (0.71 to 1.20$)$ \\
\hline $12-59$ & $131(50 \%)$ & $700(46 \%)$ & \\
\hline \multicolumn{4}{|l|}{ History and signs } \\
\hline Fever & $253(96 \%)$ & $1421(94 \%)$ & 1.63 (0.89 to 2.98) \\
\hline Cough & $260(99 \%)$ & $1498(99 \%)$ & 1.18 (0.41 to 3.43$)$ \\
\hline Difficult breathing & $234(89 \%)$ & $1388(91 \%)$ & 0.80 (0.56 to 1.13$)$ \\
\hline Vomiting & $36(14 \%)$ & $188(12 \%)$ & 1.10 (0.80 to 1.52$)$ \\
\hline Diarrhoea & $34(13 \%)$ & $156(10 \%)$ & 1.24 (0.90 to 1.73$)$ \\
\hline Past history of wheeze & $17(7 \%)$ & $73(5 \%)$ & 1.30 (0.83 to 2.02) \\
\hline \multicolumn{4}{|l|}{ Duration of illness: } \\
\hline 3 days & $187(71 \%)$ & $1135(75 \%)$ & \\
\hline$>3$ days & $76(29 \%)$ & $384(25 \%)$ & 1.17 (0.91 to 1.49) \\
\hline Presence of wheeze & $65(25 \%)$ & $350(23 \%)$ & 1.08 (0.84 to 1.40$)$ \\
\hline \multicolumn{4}{|l|}{ Season } \\
\hline October to March & $188(72 \%)$ & $1188(78 \%)$ & 0.74 (0.58 to 0.94$)$ \\
\hline
\end{tabular}

logical changes, and stayed in hospital longer. ${ }^{21}$ Current WHO guidelines do not include fever as a sign for classifying pneumonia or assessing prognosis. The value of this clinical sign for identifying those children who would benefit from treatment with antibiotics should be assessed.

In our study most children with wheeze had a normal chest radiograph. Wheeze is associated with respiratory syncytial virus $^{7}$ and antibiotics in children infected with this virus are of little benefit. ${ }^{722}$ The current generic integrated management of childhood illnesses guidelines do not include a separate algorithm for children with wheeze, although some countries have adapted these guidelines to include management of wheezing. The guidelines recommend giving two doses of rapid acting bronchodilator to children with wheeze before reassessing them for prescription of antibiotics. All children who do not respond to two cycles of inhaled bronchodilator are classified as having pneumonia and given an antibiotic. ${ }^{2}$ Children with bronchiolitis do not respond well to bronchodilators, ${ }^{23}$ therefore, using current WHO guidelines children with wheeze may still be given antibiotics unnecessarily. It is important to improve the assessment and management of children with wheeze. This will also help increase the specificity of diagnosing pneumonia and improve the rational use of antibiotics.

\section{Conclusion}

Most children diagnosed with non-severe pneumonia on the basis of fast breathing alone had normal chest radiographs and many of them may not have had bacterial pneumonia. The clinical assessment of pneumonia needs to be more specific. In addition, better and cheaper technology is needed to identify bacteria and other agents that cause pneumonia. This will help reduce the pressure on the development of resistance to antimicrobials by rationalising the use of antibiotics.

Thanks to Ehsan Latif, Saira Ansari, Sajid Farouki (Children's Hospital, Islamabad); Asifa Murtaza, Shahzad Munir (Federal Government Services Hospital, Islamabad); Sajid Maqbool, Sajid Randhawa (The Children's Hospital and Sheikh Zayed Hospital, Lahore); Imran Iqbal, Sobia Riaz (Nishtar Hospital, Multan); Zafarullah Kundi, Shazia Aslam, Rai Asghar (Rawalpindi General Hospital, Rawalpindi).

Contributors: TH helped develop the protocol, implement and monitor the study, analyse the data, and write the manuscript. YBN helped implement and monitor the study, analyse the data, and write the manuscript. SAQ helped develop the protocol, monitor the study, analyse the data, and write the manuscript. SFK and MR read all the chest radiographs. SZ read all the disputed radiographs. SAM helped write the manuscript. TH is guarantor.

\section{What is already known on this topic}

Chest radiographs are the best method for diagnosing pneumonia, but they are often not available in developing countries

World Health Organization guidelines therefore use fast breathing alone for the diagnosis of non-severe pneumonia, but fast breathing can have causes other than pneumonia

\section{What this study adds}

Most children diagnosed with non-severe pneumonia on the basis of fast breathing alone have normal chest radiographs, and many of these children may receive antibiotics unnecessarily

WHO definitions of pneumonia need to be more specific 


\section{Research}

Funding: Department of Child and Adolescent Health and Development, World Health Organization (WHO), Geneva.

Competing interests: None declared.

Ethical approval: Institutional ethical review boards of Pakistan Institute of Medical Sciences, Islamabad, Pakistan, and the secretariat committee on research involving human subjects, World Health Organization, Geneva.

1 Williams BG, Gouws E, Boschi-Pinto C, Bryce J, Dye C. Estimates of worldwide distribution of child deaths from acute respiratory infections. Lancet Infect Dis 2002;2:25-32

2 World Health Organization. Acute respiratory infections in children: case management in small hospitals in developing countries. WHO/ARI/90, 5th ed. Geneva: WHO, 1990.

3 Sazawal S, Black RE. Effect of pneumonia case management on mortality in neonates, infants and preschool children: a meta-analysis of community-based trials. Lancet Infect Dis 2003;3:547-56

4 Campbell H, Byass P, Lamount AC, Forgie IM, O'Neill KP, Lloyd Evans N, et al. Assessment of clinical criteria for identification of severe acute lower respiratory tract infections in children. Lancet 1989;1:297-9.

5 Campbell H, Byass P, Greenwood BM. Simple clinical signs for diagnosis of acute respiratory infections [letter]. Lancet 1988;2:742-3.

6 Cherian T, John TJ, Simoes E, Steinhoff MC, John M. Evaluation of simple clinical signs for the diagnosis of acute lower respiratory tract infection. Lancet 1988;2:125-8.

7 Tupasi TE, Lucero MG, Magdangal DM, Mangubat NV, Sunico ES, Torres CU, et al. Etiology of acute lower respiratory tract infection in children from Alabang, Metro Manila. Reu Infect Dis 1990;12:S929-39.

8 Shann F. Etiology of severe pneumonia in children in developing countries. Pediatr Infect Dis 1986;5:247-52.

9 World Health Organization. The overlap in the clinical presentation and treatment of malaria and pneumonia in children: report of a meeting Geneva, 8 April 1991. Geneva: WHO, $1992 . \quad$ www.who.int/child-adolescent-health/New_Publications/ CHILD HEALTH/WHO ARI 92.23.htm (last accessed 4 Aug 2006).

10 National Institutes of Health. Pocket guide for asthma management and prevention. Global initiative for asthma (GINA). NIH publication 96-3659B. Bethesda: National Institutes of Health, November 1998.

11 Ghafoor A, Nasreen NK, Ishaq Z, Zaidi SZ, Anwar F, Burney MI, et al. Diagnosis of acute lower respiratory infections in children in Rawalpindi and Islamabad, Pakistan. Rev Infect Dis 1990;12(suppl 8):S907-14.

12 Dempsey TJO, Laurence BE, McArdle TF, Todd JE, Lamont AC, Greenwood BM. The effect of temperature reduction on respiratory rate in febrile illnesses. Arch Dis Child 1993;68:492-5.

13 Morley CJ, Thornton AJ, Fowler MA, Cole TJ, Hewson PH. Respiratory rate and severity of illness in babies under 6 months old. Arch Dis Child 1990;65:834-7.

14 Morley CJ, Thornton AJ, Cole TJ, Hewson PH. Interpreting the symptoms and signs of illness in infants. In: David T, ed. Recent advances in paediatrics. London: Churchill illness in infants. In: David
Livingstone, 1991:137-55.
15 MASCOT Study Group. Clinical efficacy of three days versus five days oral amoxicillin for treatment of childhood pneumonia: a multi-centre double blind clinical trial in Pakistan. Lancet 2002;360:835-41

16 World Health Organization. Programme for the control of acute respiratory infections. Report of a meeting of the radiology working group, Geneva 27-28 October 1989. Geneva: WHO, 1990, WHO/ARI/90.13.

17 World Health Organization. Pneumonia and related infections in young children. An annotated selective bibliography. Programme for control of ARI. Geneva: WHO, 1991;1:71.

18 Stickler GB, Hoffman AD, Taylor WE. Problems in the clinical and roentgenographic diagnosis of pneumonia in young children. Clin Pediatr 1984;23:398-9.

19 Friis B, Eiken M, Hornsleth A, Jensen A. Chest X-ray appearances in pneumonia and bronchiolitis. Correlation to virological diagnosis and bacterial findings. Acta Paediatr Scand 1990;79:219-25.

20 Qazi SA, Rehman GN, Khan MA. Standard management of acute respiratory infection in a children's hospital in Pakistan: impact of antibiotic use and case fatality. Bull WHO 1996;74:501-7.

21 El-Radhi AS, Barry W, Patel S. Association of fever and severe clinical course in bronchiolitis. Arch Dis Child 1999;81:231-4.

22 Friis B, Andersen P, Brenoe E, Hornsleth A, Jensen A, Knudsen FU, et al. Antibiotic treatment of pneumonia and bronchiolitis. A prospective randomized study. Arch Dis Child 1984;59:1038-45.

23 Kellner JD, Ohlsson A, Gadomski AM, Wang EE. Bronchodilators for bronchiolitis. Cochrane Database Syst Rev 2000;(2):CD001266.

(Accepted 5 July 2006)

doi $10.1136 /$ bmj. 38915.673322 .80

ARI Research Cell, Children's Hospital, Pakistan Institute of Medical Sciences, Islamabad, Pakistan

Tabish Hazir principal investigator

Yasir Bin Nisar research administrator

Department of Radiology, Pakistan Institute of Medical Sciences

Shazia F Khan associate professor

Mujahid Raza assistant professor

Shehla Zameer assistant professor

Department of Child and Adolescent Health and Development, World Health

Organization, Geneva, Switzerland

Shamim A Qazi medical officer

Department of Paediatrics, Aga Khan University Hospital, Karachi, Pakistan

Syed Asif Masood medical officer

Correspondence to: T Hazir arichi99@arireseach.edu.pk 